Review

\title{
A finite element method based on capturing operator applied to wave propagation modeling
}

\author{
Eduardo Gomes Dutra Do Carmo ${ }^{\mathrm{a}}$, Cid Da Silva Garcia Monteiro ${ }^{\mathrm{b}, *}$, Webe João Mansur ${ }^{\mathrm{b}}$ \\ ${ }^{a}$ COPPE/UFRJ - Post-Graduate Institute Alberto Luiz Coimbra of the Federal University of Rio de Janeiro, Nuclear Engineering Department, Centro de Tecnologia, \\ Bloco G sala G-206, Cidade Universitária, Ilha do Fundão, 21945-970 Rio de Janeiro, RJ, Brazil \\ ${ }^{\mathrm{b}}$ LAMEMO-COPPE/UFRJ - Post-Graduate Institute Alberto Luiz Coimbra of the Federal University of Rio de Janeiro, Civil Engineering Department, Centro de Tecnologia, \\ Bloco B sala B-101, Cidade Universitária, Ilha do Fundão, 21945-970 Rio de Janeiro, RJ, Brazil
}

\section{A R T I C L E I N F O}

\section{Article history:}

Received 19 November 2010

Received in revised form 10 September 2011

Accepted 3 October 2011

Available online 8 October 2011

\section{Keywords:}

Finite element method

Discontinuity-capturing operators

Elastodynamics

Stabilized methods

\begin{abstract}
A B S T R A C T
This paper presents a methodology for the development of discontinuity-capturing operators for general elastodynamics. These operators are indicated for problems with sharp gradients in the space and in the time. The development here presented is based on the methodology for obtaining discontinuity-capturing operators developed by Dutra do Carmo and Galeão (1986) for diffusion-convection problems and is inspired in the works presented by Hughes and Hulbert (1988 and 1990). It is shown that their operator belongs to the families of operators developed here. The formulation is applied to one-dimensional and two-dimensional problems. The results show that the method produces better results than classic methods for the one dimensional case and presents robust performance for the two-dimensional case.
\end{abstract}

(c) 2011 Elsevier B.V. All rights reserved.

\section{Contents}

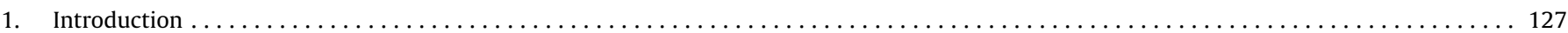

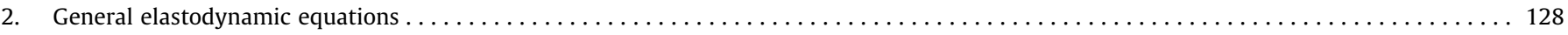

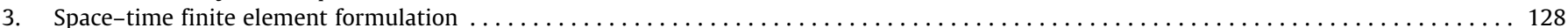

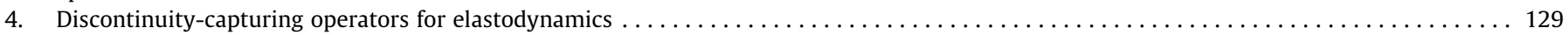

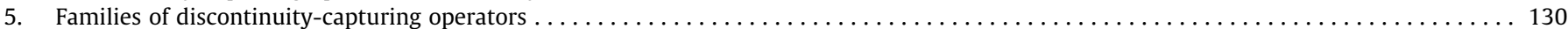

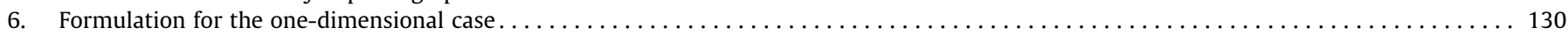

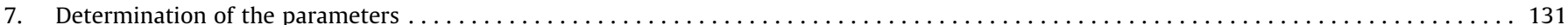

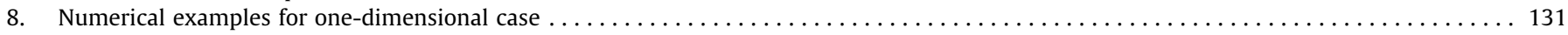

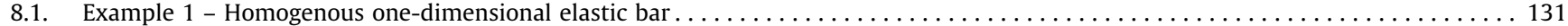

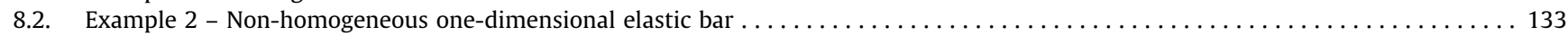

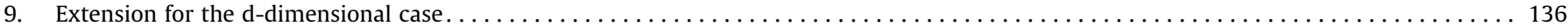

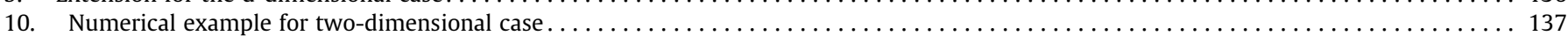

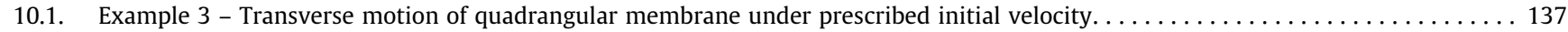

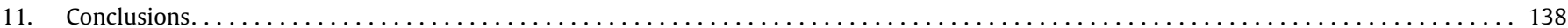

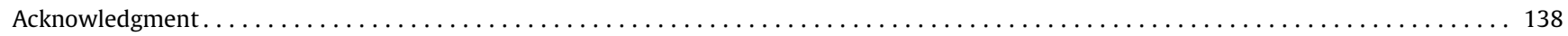

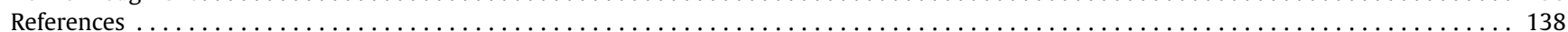

\section{Introduction}

Mathematical modeling of physical problems, static or dynamic, linear or non-linear, generally results in a system of partial

\footnotetext{
* Corresponding author. Tel.: +55 212562 7382; fax: +55 2125628464 .

E-mail addresses: egdcarmo@hotmail.com (E.G. Dutra Do Carmo),csgm25@ yahoo.com.br (C. Da Silva Garcia Monteiro), webe@coc.ufrj.br (W.J. Mansur).
}

differential equations (PDE) that must be resolved analytically or numerically to obtain the solution. In the specific case of dynamic problems, algorithms using discretization in the time are routinely used to find solutions dependent of the time. Among the various numerical methods used to solve such problems, the finite difference method (FDM) has a high computational performance, while the finite element method (FEM) is versatile and robust. 
The analysis of structural dynamics problems is usually carried out by the so called semidiscrete methods, where the FEM is used to model the spatial domain and the FDM is used to integrate on the time. Semidiscrete methods are effective for computing smooth responses, however, their performance to model problems with sharp gradients or discontinuities is not satisfactory.

Alternatively to semidiscrete methods, the use of the FEM to represent both space and time domains, was first proposed in [1-3]. Space-time finite elements can represent better the solution of a problem than semidiscrete methods. However, both present difficulties in the presence of discontinuities as described in references $[4,5]$.

It was developed in [6] a space-time finite element formulation for elastodynamics where the solution and it's derivative in the time are discontinuous between of two consecutive time intervals. Capturing operators were included in this formulation to capture the discontinuities.

Since then many works have been developed to represent discontinuities or sharp gradients for the FD and FE methods. A general review can be seen in $[7,8]$ and a very instructive review concerning spurious oscillations for convection-diffusion equations can be seen in [9].

In [10] it was presented another time discontinuous Galerkin method where time and space are decoupled.

The objective of the present work is to extend the methodology developed in $[11,12]$ to elastodynamics. This paper is organized as follows.

In Section 2 we present the basic equations of the elastodynamics. Section 3 is devoted to the time discontinuous Galerkin formulation. In Sections 4 and 5 we present the general methodology to obtain the discontinuity capturing operators. In Sections 6 and 7 we show how to obtain the capturing operator for wave propagation in one-dimensional spaces and determine the parameters of the proposed operator. Section 8 presents two numerical examples for one-dimensional case. In Section 9 we propose a possible extension of the discontinuity capturing operator to the $d$-dimensional case, and the Section 10 presents one numerical example for two-dimensional case using the extension proposed in the previous section. Finally we present the conclusions in Section 11

\section{General elastodynamic equations}

Let $\Omega \subset R^{d}$, where $d$ is space dimension, be an elastic linear solid with boundary Lipshitz continuous $\Gamma=\partial \Omega=\Gamma_{g} \cup \Gamma_{h}$ and being meas $\left(\Gamma_{g} \cap \Gamma_{h}\right)=0$, where meas(.) denotes Lebesgue positive measure. Elastic wave propagation in solids is governed by the following second order hyperbolic partial differential equation

$\rho \ddot{\mathbf{u}}-\nabla \cdot \boldsymbol{\sigma}(\nabla \mathbf{u})-\mathbf{f}=0 \quad$ on $Q=\Omega \times[0, T]$,

where the stress $\boldsymbol{\sigma}(\nabla \mathbf{u})$ is given by the generalized Hooke's law:

$\boldsymbol{\sigma}(\nabla \mathbf{u})=\mathbf{C} . \nabla \mathbf{u}$

where $\mathbf{C}$ is a fourth-order tensor whose components are the elastic coefficients.

Therefore, the component $\sigma_{i j}$ is given as follows

$\sigma_{i j}=\sum_{l=1}^{d} \sum_{k=1}^{d} C_{i j k l} \frac{\partial u_{k}}{\partial x_{l}}$,

with boundary conditions and initial conditions given below

$$
\begin{aligned}
& \mathbf{u}=\mathbf{g} \quad \text { on } \Gamma_{g} \times[0, T], \\
& \mathbf{n} \cdot \boldsymbol{\sigma}(\nabla \mathbf{u})=\mathbf{h} \quad \text { on } \Gamma_{h} \times[0, T], \\
& \mathbf{u}(\mathbf{x}, 0)=\mathbf{u}_{0}(\mathbf{x}), \\
& \dot{\mathbf{u}}(\mathbf{x}, 0)=\mathbf{v}_{0}(\mathbf{x}),
\end{aligned}
$$

where $\mathbf{g}$ and $\mathbf{h}$ represent respectively the prescribed boundaries displacement and traction, $\mathbf{n}$ denotes the unit outward vector normal to $\Gamma$, $\mathbf{u}$ denotes the displacement vector, $\mathbf{u}$ denotes the differentiation of $\mathbf{u}$ with respect to the time variable $t . T>0, \rho$ denotes the mass density and $\mathbf{u}_{0}(\mathbf{x})$ and $\mathbf{v}_{0}(\mathbf{x})$ represent respectively initial displacement and initial velocity.

\section{Space-time finite element formulation}

For $n \in\{0, \ldots, N\}$ consider the time interval $I_{n}=\left[t_{n-1}, t_{n}\right]$, the time step $\Delta t=t_{n}-t_{n-1}$, and the jump operator defined as

$$
\begin{aligned}
& \llbracket u\left(t_{n}\right) \rrbracket=u\left(t_{n}^{+}\right)-u\left(t_{n}^{-}\right), \\
& u\left(t_{n}^{+}\right)=\lim _{\varepsilon \rightarrow 0^{+}} u\left(t_{n}+\varepsilon\right), \\
& u\left(t_{n}^{-}\right)=\lim _{\varepsilon \rightarrow 0^{-}} u\left(t_{n}+\varepsilon\right) .
\end{aligned}
$$

The variational equation or weak form can be derived from a weighting residual form as given in [6,7], (see expression (8)), by considering the jump operator in the time for displacement and velocity, as follows

$$
\begin{aligned}
& \int_{t_{n-1}}^{t_{n}} \int_{\Omega} \dot{\mathbf{W}}(\mathbf{x}, t) \rho \ddot{\mathbf{u}}(\mathbf{x}, t) d \Omega d t-\int_{t_{n-1}}^{t_{n}} \int_{\Omega} \dot{\mathbf{W}}(\mathbf{x}, t) \nabla \cdot \boldsymbol{\sigma}(\nabla \mathbf{u}(\mathbf{x}, t)) d \Omega d t \\
& \quad-\int_{t_{n-1}}^{t_{n}} \int_{\Omega} \dot{\mathbf{W}}(\mathbf{x}, t) \mathbf{f}(\mathbf{x}, t) d \Omega d t+\int_{\Omega} \dot{\mathbf{W}}\left(\mathbf{x}, t_{n-1}^{+}\right) \rho \llbracket \dot{\mathbf{u}}(\mathbf{x}, t) \rrbracket d \Omega \\
& \quad+\int_{\Omega} \mathrm{W}\left(\mathbf{x}, t_{n-1}^{+}\right) \nabla \cdot \boldsymbol{\sigma}\left(\llbracket \nabla \mathbf{u}\left(\mathbf{x}, t_{n-1}\right) \rrbracket\right) d \Omega=0 .
\end{aligned}
$$

After applying integration by parts to reduce the order of the spatial operator and by considering the divergence theorem, one obtains

$$
\begin{aligned}
\int_{t_{n-1}}^{t_{n}} & \int_{\Omega} \dot{\mathbf{W}}(\mathbf{x}, t) \rho \ddot{\mathbf{u}}(\mathbf{x}, t) d \Omega d t+\int_{t_{n-1}}^{t_{n}} \int_{\Omega} \nabla \dot{\mathbf{W}}(\mathbf{x}, t) \boldsymbol{\sigma}(\nabla \mathbf{u}(\mathbf{x}, t)) d \Omega d t \\
& -\int_{t_{n-1}}^{t_{n}} \int_{\Gamma_{h}} \dot{\mathbf{W}}(\mathbf{x}, t) \mathbf{h}(\mathbf{x}, t) d t-\int_{t_{n-1}}^{t_{n}} \int_{\Omega} \dot{\mathbf{W}}(\mathbf{x}, t) \mathbf{f}(\mathbf{x}, t) d \Omega d t \\
& +\left(\int_{\Omega} \dot{\mathbf{W}}\left(\mathbf{x}, t_{n-1}^{+}\right) \rho \dot{\mathbf{u}}\left(\mathbf{x}, t_{n-1}^{+}\right) d \Omega-\int_{\Omega} \dot{\mathbf{W}}\left(\mathbf{x}, t_{n-1}^{+}\right) \rho \dot{\mathbf{u}}\left(\mathbf{x}, t_{n-1}^{-}\right) d \Omega\right) \\
& +\left(\int_{\Omega} \nabla \mathbf{W}\left(\mathbf{x}, t_{n-1}^{+}\right) \boldsymbol{\sigma}\left(\nabla \mathbf{u}\left(\mathbf{x}, t_{n-1}^{+}\right)\right) d \Omega\right. \\
& \left.-\int_{\Omega} \nabla \mathbf{W}\left(\mathbf{x}, t_{n-1}^{+}\right) \boldsymbol{\sigma}\left(\nabla \mathbf{u}\left(\mathbf{x}, t_{n-1}^{-}\right)\right) d \Omega\right)=0 .
\end{aligned}
$$

In order to work out the approximate formulation through the finite element method, consider a usual partition of the domain $\Omega$ into ne elements. For each $\Omega_{e}$ and each time interval $I_{n}$, let $P^{k}\left(Q_{n}^{e}\right)$ be the space of the polynomials of degree $\leqslant k$ in the local coordinates where $Q_{n}^{e}=\Omega_{e} \times I_{n}$. By considering $k \geqslant 2$ one has the set of the admissible approximations

$S^{h, k}=\left\{\mathbf{u}^{h} \mid \mathbf{u}^{h} \in\left(C^{0}\left(\cup_{n=1}^{N} Q_{n}\right)\right), \mathbf{u}_{e}^{h} \in P^{k}\left(Q_{n}^{e}\right), \mathbf{u}^{h}=\mathbf{g}\right.$ on $\left.\Gamma_{g} \times I\right\}$,

and the space of the admissible variations

$$
V^{h, k}=\left\{\mathbf{W}^{h} \mid \mathbf{W}^{h} \in\left(C^{0}\left(\cup_{n=1}^{N} Q_{n}\right)\right), \mathbf{W}_{e}^{h} \in P^{k}\left(Q_{n}^{e}\right), \mathbf{W}^{h}=0 \text { on } \Gamma_{g} \times I\right\},
$$

where $\mathbf{u}_{e}^{h}$ denotes the restriction of $\mathbf{u}^{h}$ to $Q_{n}^{e}$. The Time Discontinuous Galerkin formulation associated to the variational problem 
given by (9), consists of finding $\mathrm{u}^{h} \in S^{h, k}$ satisfying the variational equation

$$
A_{T D G}\left(\mathrm{u}^{h}, \mathrm{~W}^{h}\right)_{n}=F_{T D G}\left(\mathrm{~W}^{h}\right)_{n} \forall \mathrm{W}^{h} \in V^{h, k},
$$

$$
A_{T D G}\left(\mathbf{u}^{h}, \mathbf{W}^{h}\right)_{n}=\sum_{e=1}^{n e}\left[\begin{array}{c}
\int_{t_{n-1}}^{t_{n}} \int_{\Omega_{e}} \dot{\mathbf{W}}^{h}(\mathbf{x}, \boldsymbol{t}) \rho \ddot{\mathbf{u}}^{h}(\mathbf{x}, \boldsymbol{t}) d \Omega d t+ \\
\int_{t_{n-1}}^{t_{n}} \int_{\Omega_{e}} \nabla \dot{\mathbf{W}}^{h}(\mathbf{x}, \boldsymbol{t}) \boldsymbol{\sigma}\left(\nabla \mathbf{u}^{h}(\mathbf{x}, \boldsymbol{t})\right) d \Omega d t+ \\
\int_{\Omega_{e}} \mathbf{W}^{h}\left(\mathbf{x}, \boldsymbol{t}_{n-1}^{+}\right) \rho \dot{\mathbf{u}}^{h}\left(\mathbf{x}, \mathbf{t}_{n-1}^{+}\right) d \Omega+ \\
\int_{\Omega_{e}} \nabla \mathbf{W}^{h}\left(\mathbf{x}, \mathbf{t}_{n-1}^{+}\right) \boldsymbol{\sigma}\left(\nabla \mathbf{u}^{h}\left(\mathbf{x}, \mathbf{t}_{n-1}^{+}\right)\right) d \Omega
\end{array}\right],
$$

$$
F_{T D G}\left(\mathbf{W}^{h}\right)_{n}=\sum_{e=1}^{n e}\left[\begin{array}{c}
\int_{t_{n-1}}^{t_{n}} \int_{\Gamma_{h e}} \dot{\mathbf{W}}^{h}(\mathbf{x}, \boldsymbol{t}) \mathbf{h}(\mathbf{x}, \mathbf{t}) d \Omega d t- \\
\int_{t_{n-1}}^{t_{n}} \int_{\Omega_{e}} \dot{\mathbf{W}}^{h}(\mathbf{x}, \boldsymbol{t}) \mathbf{f}(\mathbf{x}, \boldsymbol{t}) d \Omega d t+ \\
\int_{\Omega_{e}} \dot{\mathbf{W}}^{h}\left(\mathbf{x}, \boldsymbol{t}_{n-1}^{+}\right) \rho \dot{\mathbf{u}}^{h}\left(\mathbf{x}, \boldsymbol{t}_{n-1}^{-}\right) d \Omega+ \\
\int_{\Omega_{e}} \nabla \mathbf{W}^{h}\left(\mathbf{x}, \boldsymbol{t}_{n-1}^{+}\right) \boldsymbol{\sigma}\left(\nabla \mathbf{u}^{h}\left(\mathbf{x}, \boldsymbol{t}_{n-1}^{-}\right)\right) d \Omega
\end{array}\right]
$$

$$
F_{\mathrm{TDG}}\left(\mathbf{W}^{h}\right)_{1}=\sum_{e=1}^{n e}\left[\begin{array}{c}
\int_{t_{n-1}}^{t_{n}} \int_{\Gamma_{h_{e}}} \dot{\mathbf{W}}^{h}(\mathbf{x}, t) \mathbf{h}(\mathbf{x}, \boldsymbol{t}) d \Omega d t- \\
\int_{t_{n-1}}^{t_{n}} \int_{\Omega_{e}} \dot{\mathbf{W}}^{h}(\mathbf{x}, \boldsymbol{t}) \mathbf{f}(\mathbf{x}, \mathbf{t}) d \Omega d t+ \\
\int_{\Omega_{e}} \dot{\mathbf{W}}^{h}\left(\mathbf{x}, \mathbf{t}_{n-1}^{+}\right) \rho \mathbf{v}_{\mathbf{0}}(\mathbf{x}) d \Omega+ \\
\int_{\Omega_{e}} \nabla \mathbf{W}^{h}\left(\mathbf{x}, \mathbf{t}_{n-1}^{+}\right) \boldsymbol{\sigma}\left(\nabla \mathbf{u}_{0}(\mathbf{x})\right) d \Omega
\end{array}\right] .
$$

\section{Discontinuity-capturing operators for elastodynamics}

In this section we present a general methodology to develop discontinuity-capturing operators for the general elastodynamic problem presented in Section 2. We consider again, an elastic body occupying a bounded region $\Omega$ contained in $R^{d}$, where $d \in\{1,2,3\}$, the stress components $\sigma_{i j}$ being given as

$\sigma_{i j}=\sum_{l=1}^{d} \sum_{k=1}^{d} C_{i j k l} \frac{\partial u_{k}}{\partial x_{l}}$

where $i, j, k, l \in\{1, \ldots, d\}$ and $C_{i j k l}$ are the elastic coefficients.

In order to extend to elastodynamics the methodology presented in [11] for diffusion-convection problem, we consider the equilibrium equations

$\rho \frac{\partial^{2} u_{i}}{\partial t^{2}}-\left[\sum_{j=1}^{d} \sum_{k=1}^{d} \sum_{l=1}^{d}\left[C_{i j k l} \frac{\partial^{2} u_{k}}{\partial x_{j} \partial x_{l}}+\frac{\partial C_{i j k l}}{\partial x_{j}} \frac{\partial u_{k}}{\partial x_{l}}\right]\right]=f_{i}(i=1, \ldots, d)$.

By following the methodology presented in [11], associated to one $\mathbf{u}^{h}$ fixed, we consider the approximate coefficients $C_{i j k l}^{h}, \frac{\partial C_{i j k l}^{h}}{\partial x_{i}}$ and $\rho_{i}^{h}$ satisfying

$\rho_{i}^{h} \frac{\partial^{2} u_{i}^{h}}{\partial t^{2}}-\left[\sum_{j=1}^{d} \sum_{k=1}^{d} \sum_{l=1}^{d}\left[\delta_{i j k l} C_{i j k l}^{h} \frac{\partial^{2} u_{k}^{h}}{\partial x_{j} \partial x_{l}}+\delta_{i j k l}^{j} \frac{\partial C_{i j k l}^{h}}{\partial x_{j}} \frac{\partial u_{k}^{h}}{\partial x_{l}}\right]\right]$

$$
-f_{i}=0(i=1, \ldots, d) \text {, }
$$

where

$\delta_{i j k l}=\left\{\begin{array}{ll}1, & \text { if } C_{i j k l} \neq 0 \\ 0, & \text { otherwise }\end{array}\right.$ and $\delta_{i j k l}^{j}= \begin{cases}1, & \text { if } \frac{\partial C_{i j k l}}{\partial x_{j}} \neq 0 \\ 0, & \text { otherwise. }\end{cases}$

Therefore, given an approximate solution $\mathbf{u}^{h}$, the goal is to find the approximate coefficients, $C_{i j k l}^{h}, \frac{\partial C_{i j k l}^{h}}{\partial x_{i}}$ and $\rho_{i}^{h}$ satisfying (18) and as close to $C_{i j k l}, \frac{\partial C_{i j k l}}{\partial x_{i}}$ and $\rho_{i}$ as possible. To achieve this goal, we consider the functional $J^{*}$, defined as

$$
\begin{aligned}
J^{*}= & \sum_{i}^{d}\left\{\sum_{j}^{d} \sum_{l}^{d} \sum_{k}^{d} \frac{\left[C_{i j k l}^{h}-C_{i j k l}\right]^{2}}{2}+\frac{\theta_{i}}{2}\left[\frac{\partial C_{i j k l}^{h}}{\partial x_{j}}-\frac{\partial C_{i j k l}}{\partial x_{j}}\right]^{2}\right\} \\
& +\frac{\gamma_{i}}{2}\left[\rho_{i}^{h}-\rho\right]^{2},
\end{aligned}
$$

where $\theta_{i}$ and $\gamma_{i}$ are dimensional parameters so that the terms of the equation above can have the same dimension and are defined as follows

$\gamma_{i}=\left(\frac{C_{i i i i}}{\rho}\right)^{2}$,

$\theta_{i}=\left(\operatorname{Cour}_{i}\right)^{2}\left(h_{e, i}\right)^{2}$,

where Cour $_{i}$ is the Courant number on the direction $i$, and $h_{e, i}$ is the characteristic length on the direction $i$, both will be defined later on in this section.

Our objective is to minimize the functional $J^{*}$ subjected to the restriction given by (18). The problem of minimization of the functional $J^{*}$ satisfying the restrictions (18) is equivalent to minimize the functional $J$, defined as

$J=\sum_{i}^{d}\left[\begin{array}{c}\left\{\sum_{j}^{d} \sum_{l}^{d} \sum_{k}^{d} \frac{\left[c_{i j k l}^{h}-C_{i j k l}\right]^{2}}{2}+\frac{\theta_{i}}{2}\left[\frac{\partial c_{i j k l}^{h}}{\partial x_{j}}-\frac{\partial C_{i j k l}}{\partial x_{j}}\right]^{2}\right\}+\frac{\gamma_{i}}{2}\left[\rho_{i}^{h}-\rho\right]^{2}+ \\ \lambda_{i}\left[-\sum_{j}^{d} \sum_{k}^{d} \sum_{l}^{d}\left[C_{i j k l}^{h} \delta_{i j k l}\left(\frac{\partial^{2} u_{k}^{h}}{\partial x_{j} \partial x_{l}}\right)+\delta_{i j k l}^{j}\left(\frac{\partial c_{i j k l}^{h}}{\partial x_{j}}\right)\left(\frac{\partial u_{k}^{h}}{\partial x_{l}}\right)\right]+\rho_{i}^{h} \frac{\partial^{2} u_{i}^{h}}{\partial t^{2}}-f_{i}\right]\end{array}\right]$,

where $\lambda_{i}$ are Lagrange Multipliers.

By minimizing the $J$ functional with respect to $C_{i j k l}^{h}, \frac{\partial C_{i j k l}^{h}}{\partial x_{i}}, \rho_{i}^{h}$ and $\lambda_{i}$, for one $\mathbf{u}^{h}$ fixed, and by performing subsequently some manipulations and by considering the vector

$\mathbf{U}^{i}\left(\mathbf{u}^{h}\right)=\left(\mathbf{U}^{i, 1}\left(\mathbf{u}^{h}\right), \mathbf{U}^{i, 2}\left(\mathbf{u}^{h}\right), \frac{1}{\sqrt{\gamma_{i}}} \frac{\partial^{2} u_{i}^{h}}{\partial t^{2}}\right)$,

where

$\mathbf{U}^{i, 1}\left(\mathbf{u}^{h}\right)=\left(\delta_{i 111} \frac{\partial^{2} u_{1}^{h}}{\partial x_{1}^{2}}, \ldots, \delta_{i j k l} \frac{\partial^{2} u_{k}^{h}}{\partial x_{j} \partial x_{l}}, \ldots, \delta_{i d d d} \frac{\partial^{2} u_{d}^{h}}{\partial x_{d}^{2}}\right)$,

$\mathbf{U}^{i, 2}\left(\mathbf{u}^{h}\right)=\left(\frac{\delta_{i 111}^{1}}{\sqrt{\theta_{i}}} \frac{\partial u_{1}^{h}}{\partial x_{1}}, \ldots, \frac{\delta_{i j k l}^{j}}{\sqrt{\theta_{i}}} \frac{\partial u_{k}^{h}}{\partial x_{l}}, \ldots, \frac{\delta_{i d d d}^{d}}{\sqrt{\theta_{i}}} \frac{\partial u_{d}^{h}}{\partial x_{d}}\right)$,

one can obtain the Lagrange multipliers in the compact format

$\lambda_{i}=\frac{R_{i}\left(\mathbf{u}^{h}\right)}{\left\|\mathbf{U}^{i}\left(\mathbf{u}^{h}\right)\right\|^{2}}(i=1, \ldots, d)$

where

$R_{i}\left(\mathbf{u}^{h}\right)=-\left[\sum_{j}^{n} \sum_{k}^{n} \sum_{l}^{n}\left[C_{i j k l}\left(\frac{\partial^{2} u_{k}^{h}}{\partial x_{j} \partial x_{l}}\right)+\frac{\partial C_{i j k l}}{\partial x_{j}} \frac{\partial u_{k}^{h}}{\partial x_{l}}\right]\right]+\rho \frac{\partial^{2} u_{i}^{h}}{\partial t^{2}}$

$$
-f_{i}(i=1, \ldots, d) \text {. }
$$

By defining the vector of disturbance or error vector

$\mathrm{V}_{p}^{h, i}\left(\mathrm{u}^{h}\right)=\left(\mathrm{V}_{p}^{h, i, 1}, \mathrm{~V}_{p}^{h, i, 2}, \sqrt{\gamma_{i}}\left(\rho-\rho_{i}^{h}\right)\right)$ 
where

$$
\begin{aligned}
\mathrm{V}_{p}^{h, i, 1}\left(\mathrm{u}^{h}\right)= & \left(\left(C_{i 111}^{h}-C_{i 111}\right), \ldots,\left(C_{i j k l}^{h}-C_{i j k l}\right), \ldots,\left(C_{i d d d}^{h}-C_{i d d d}\right)\right) \\
\mathrm{V}_{p}^{h, i, 2}\left(\mathrm{u}^{h}\right)= & \left(\sqrt{\theta_{i}}\left(\frac{\partial C_{i 111}^{h}}{\partial x_{1}}-\frac{\partial C_{i 111}}{\partial x_{1}}\right), \ldots,\right. \\
& \left.\sqrt{\theta_{i}}\left(\frac{\partial C_{i j k l}^{h}}{\partial x_{j}}-\frac{\partial C_{i j k l}}{\partial x_{j}}\right), \ldots, \sqrt{\theta_{i}}\left(\frac{\partial C_{i d d d}^{h}}{\partial x_{d}}-\frac{\partial C_{i d d d}}{\partial x_{d}}\right)\right),
\end{aligned}
$$

with $(i=1, \ldots, d)$, and from Eqs. (22a-c), (23a-b) and (24a-c) we obtain

$\mathrm{V}_{p}^{h, i}\left(\mathrm{u}^{h}\right)=\frac{R_{i}\left(\mathrm{u}^{h}\right) \mathrm{U}^{i}\left(\mathrm{u}^{h}\right)}{\left\|\mathrm{U}^{i}\left(\mathrm{u}^{h}\right)\right\|^{2}}(i=1, \ldots, d)$,

$\widehat{\mathrm{V}}_{p}^{h, i}\left(\mathrm{u}^{h}\right)=\frac{\mathrm{V}_{p}^{h, i}\left(\mathrm{u}^{h}\right)}{\left\|\mathrm{V}_{p}^{h, i}\left(\mathrm{u}^{h}\right)\right\|}=\frac{R_{i}\left(\mathrm{u}^{h}\right) \mathrm{U}^{i}\left(\mathrm{u}^{h}\right)}{\left|R_{i}\left(\mathrm{u}^{h}\right)\right|\left\|\mathrm{U}^{i}\left(\mathrm{u}^{h}\right)\right\|}(i=1, \ldots, d)$

Associated to $\mathbf{U}^{i}\left(\mathbf{u}^{h}\right)$ we consider the vector $\mathbf{U}^{i, l o c}\left(\mathbf{u}^{h}\right)$ in the local coordinates,

$\mathbf{U}^{i, l o c}\left(\mathbf{u}^{h}\right)=\left(\delta_{i 111} \frac{\partial^{2} u_{1}^{h}}{\partial \xi_{1}^{2}} \ldots, \delta_{i j k l} \frac{\partial^{2} u_{k}^{h}}{\partial \xi_{j} \partial \xi_{l}}, \ldots, \delta_{i d d d} \frac{\partial^{2} u_{d}^{h}}{\partial \xi_{d}^{2}}, \frac{\partial^{2} u_{i}^{h}}{\partial \xi_{0}^{2}}\right)$,

$(i=1, \ldots, d)$,

where $\left(\xi_{1}, \ldots, \xi_{d}\right)$ are the dimensionless coordinates of the element related to the physical or global coordinates and $\xi_{0}$ is a dimensionless coordinate related to the time.

In order to obtain the Petrov-Galerkin disturbance necessary for building a discontinuity-capturing operator family, we use the expressions (24a)-(24c), (25)-(27) for introducing the functions

$h^{l o c}\left(\mathbf{u}^{h}\right)=\left[\frac{\mathrm{U}^{i, l o c}\left(\mathrm{u}^{h}\right)}{\left\|\mathrm{U}^{i}\left(\mathbf{u}^{h}\right)\right\|}\right]^{1 / 2}$,

$\tau_{i}\left(\mathbf{u}^{h}, \alpha_{i}\right)= \begin{cases}{\left[\frac{1}{\left\|\mathbf{U}^{i}\left(\mathbf{u}^{h}\right)\right\|}\left|\frac{R_{i}\left(\mathbf{u}^{h}\right)}{\rho\left(\gamma_{i}\right)^{1 / 2}}\right|\right]^{\alpha_{i}},} & \text { if }\left\|\mathbf{U}^{i}\left(\mathbf{u}^{h}\right)\right\|>0 \\ 0, & \text { if }\left\|\mathbf{U}^{i}\left(\mathbf{u}^{h}\right)\right\|=0\end{cases}$

$\operatorname{Cour}_{i}=\frac{\left(\gamma_{i}\right)^{1 / 4} \Delta t}{h_{e, i}}$,

$h_{e, i}=\operatorname{Sup}_{x, y \in \Omega_{e}}\left|x_{i}-y_{i}\right|$,

where Sup denotes Supremum and $\alpha_{i}=\alpha_{i}\left(\right.$ Cour $\left._{i}\right) \geqslant 0$.

Our Petrov-Galerkin disturbance is then given by

$p_{i}\left(\mathbf{u}^{h}, \mathbf{W}^{h}\right)=\Delta t \rho \gamma_{i} \tau_{i}\left(\mathbf{u}^{h}, \alpha_{i}\right)\left[\frac{\widehat{\mathbf{V}}_{p}^{h, i}\left(\mathbf{u}^{h}\right)}{\rho\left(\gamma_{i}\right)^{1 / 2}} \cdot \mathbf{W}_{i}\left(\mathbf{W}^{h}\right)\right]$,

where $\mathbf{W}_{i}\left(\mathbf{W}^{h}\right) \in R^{d} \quad \forall \mathbf{W}^{h} \in V^{h, k}$.

Finally, a discontinuity-capturing operator family related to the degree of freedom $i$ can be obtained by integrating over the spacetime domain the product between the residue $R_{i}\left(\mathbf{u}^{h}\right)$ and the Petrov-Galerkin disturbance $p_{i}\left(\mathbf{u}^{h}, \mathbf{W}^{h}\right)$ as follows

$$
\begin{aligned}
A_{C D}\left(\mathbf{u}^{h}, \mathbf{W}^{h}\right)_{n}= & \sum_{i=1}^{d} \sum_{e=1}^{n e} \int_{t_{n-1}}^{t_{n}} \int_{\Omega_{e}}^{\Delta t} \rho \gamma_{i} \tau_{i}\left(\mathbf{u}^{h}, \alpha_{i}+1\right) \\
& \times\left[\mathbf{U}^{i}\left(\mathbf{u}^{h}\right) \cdot \mathbf{W}_{i}\left(\mathbf{W}^{h}\right)\right] d \Omega d t .
\end{aligned}
$$

We notice that to each pair $\left(\mathbf{W}_{i}\left(\mathbf{W}^{h}\right), \alpha_{i}\right)$ corresponds a capturing operator.

\section{Families of discontinuity-capturing operators}

Families of discontinuity-capturing operators for elastodynamics can be obtained by defining suitable $\mathbf{W}_{i}\left(W^{h}\right)$. For instance, consider the following form

$$
\begin{aligned}
\mathbf{W}_{i}\left(\mathbf{W}^{h}\right)= & \beta_{i}\left(\operatorname{Cour}_{i}, h^{l o c}\left(\mathbf{u}^{h}\right), \alpha_{i}\left(\operatorname{Cour}_{i}\right)\right) \cdot \frac{1}{\left(h^{l o c}\left(\mathbf{u}^{h}\right)\right)^{4}} \\
& \times \frac{\left[\mathrm{U}^{i, l o c}\left(\mathbf{u}^{h}\right) \cdot \mathrm{U}^{i, l o c}\left(\mathbf{W}^{h}\right)\right]}{\mathrm{U}^{i}\left(\mathbf{u}^{h}\right) \cdot \mathrm{U}^{i}\left(\mathbf{W}^{h}\right)} \mathrm{U}^{i}\left(\mathbf{W}^{h}\right)
\end{aligned}
$$

where $\beta_{i}$ is a non-negative dimensionless function.

A specific family of operators can be obtained by making the $\alpha_{i}$ $\left(\right.$ Cour $_{i}$ ) equal to a real number independent of the Courant number as follows

$\alpha_{i}\left(\operatorname{Cour}_{i}\right)=\bar{\alpha} \geqslant 0 \forall i$ and $\forall \operatorname{Cour}_{i}$.

By considering $\mathbf{W}_{i}\left(\mathbf{W}^{h}\right)$ as given by the Eq. (34), $\tau_{i}\left(\mathbf{u}^{h}, \alpha_{i}+1\right)$ as given in the Eq. (29) and $\bar{\alpha}$ constant, we can obtain the class of capturing operator characterized by the Eq. (36) below

$$
\begin{aligned}
A_{C D}^{e}\left(\mathbf{u}^{h}, \mathbf{W}^{h}\right)= & \sum_{i} \int_{t_{n-1}}^{t_{n}} \int_{\Omega_{e}} \Delta t \frac{\left|R_{i}\left(\mathbf{u}^{h}\right)\right|^{\bar{\alpha}+1}}{\rho^{\bar{\alpha}} \gamma_{i}^{(\bar{\alpha}-1) / 2}} \\
& \times \frac{\left\|\mathbf{U}^{i}\left(\mathbf{u}^{h}\right)\right\|^{1-\bar{\alpha}}}{\left\|\mathbf{U}^{i, l o c}\left(\mathbf{u}^{h}\right)\right\|^{2}} \beta_{i}\left[\mathbf{U}^{i, l o c}\left(\mathbf{u}^{h}\right) \cdot \mathbf{U}^{i, l o c}\left(\mathbf{W}^{h}\right)\right] d \Omega d t,
\end{aligned}
$$

where $\beta_{i}=\beta_{i}\left(\operatorname{Cour}_{i}, h^{\text {loc }}\left(\mathbf{u}^{h}\right), \bar{\alpha}\right)$.

In this class many discontinuity-capturing operators can be obtained by choosing $\bar{\alpha}$ and $\beta_{i}$. The operator presented in [7,8] for the one-dimensional case, can be obtained with $\bar{\alpha}=1$ and $\beta_{1}=\left(\Delta t /\left(4 \sqrt{1+\text { Cour }^{4}}\right)\right) / \Delta t$.

\section{Formulation for the one-dimensional case}

In this section we present the expressions concerning the discontinuity-capturing operator for the one-dimensional elastic case and subsequently present a methodology to determine the operator parameters.

Consider the wave propagation problem on a one-dimensional bar with constant coefficients, given by Eq. (37)

$\frac{1}{\gamma^{1 / 2}} \frac{\partial^{2} u}{\partial t^{2}}-\frac{\partial^{2} u}{\partial x^{2}}=f(t)$

The discontinuity-capturing operator for this one-dimensional case is

$A_{C D}\left(\mathbf{u}^{h}, \mathbf{W}^{h}\right)_{n}=\sum_{e=1}^{n e} \int_{t_{n-1}}^{t_{n}} \int_{\Omega_{e}}^{\Delta t} \rho \gamma \tau\left(\mathbf{u}^{h}, \bar{\alpha}+1\right)\left[\mathbf{U}\left(\mathbf{u}^{h}\right) \cdot \mathbf{W}\left(\mathbf{W}^{h}\right)\right] d \Omega d t$,

where the vector $\mathbf{U}\left(\mathbf{u}^{h}\right)$ is given by

$\mathbf{U}\left(\mathbf{u}^{h}\right)=\left(\frac{\partial^{2} u^{h}}{\partial x^{2}}, \frac{1}{\gamma^{1 / 2}} \frac{\partial^{2} u^{h}}{\partial t^{2}}\right)$

and the weight vector $\mathbf{W}\left(W^{h}\right)$ was chosen as being

$\mathbf{W}\left(W^{h}\right)=\left\{\begin{array}{ll}\mathbf{W}_{-}\left(W^{h}\right)=\mu^{-}(\operatorname{Cour})\left(\frac{\partial^{2} W^{h}}{\partial x^{2}}, \frac{\operatorname{Cour}^{4}}{\gamma^{1 / 2}} \ddot{W}^{h}\right), & \text { if Cour }<1 \\ \mathbf{W}_{+}\left(W^{h}\right)=\mu^{+}(\operatorname{Cour})\left(\operatorname{Cour}^{-4} \frac{\partial^{2} W^{h}}{\partial x^{2}}, \frac{1}{\gamma^{1 / 2}} \ddot{W}^{h}\right), & \text { if Cour } \geqslant 1\end{array}\right.$,

where $\bar{\alpha}$ is a real number to be determined, $\gamma^{1 / 2}=c^{2}$ ( $c$ being the wave propagation velocity) and $\mu^{+}$(Cour) and $\mu^{-}$(Cour) are 


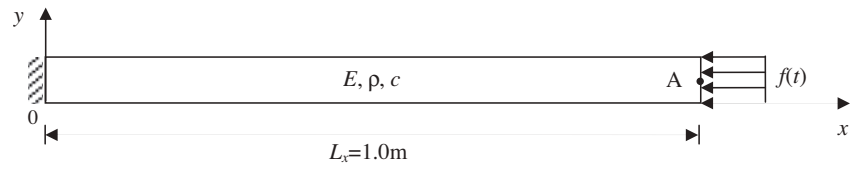

Fig. 1. Homogeneous elastic bar used to determine the discontinuity capturing parameters.

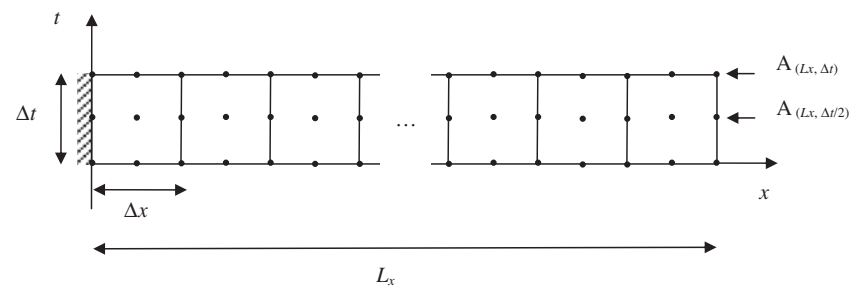

Fig. 2. Space-time slab associated to the bar.

dimensionless functions dependent of the Courant number. In the next section we present how the parameters for the one-dimensional elastic bar are determined.

\section{Determination of the parameters}

The parameters $\bar{\alpha}, \mu^{+}$(Cour) and $\mu^{-}$(Cour) are determined via numerical experiments. The problem chosen to determine these parameters is a one-dimensional elastic bar. The bar has one end fixed and the other loaded by an axial force as shown in Fig. 1, and has the following properties: the length is $L_{x}=1 \mathrm{~m}$, the square cross section area is $0.01 \mathrm{~m}^{2}$, the mass density is $\rho=1 \mathrm{~kg} / \mathrm{m}^{3}$ and Young's modulus is $E=1 \mathrm{~N} / \mathrm{m}^{2}$. The exact solution for this problem can be found in [13].

The developed formulation uses space-time elements unlike traditional semidiscrete finite element formulations. The spacetime slab was discretized using 50 quadrilateral elements of 9 nodes, as shown in Fig. 2.

The variables used to determine the functions $\mu^{+}$(Cour), $\mu^{-}$(Cour) and the exponent $\bar{\alpha}$ are the velocity distribution along the time at the point $\mathrm{A}$ (free end of the bar) and the stress distribution along the bar at a specific time.

The experiment for the determination of the parameters $\mu^{+}$(Cour), $\mu^{-}$(Cour) and the exponent $\bar{\alpha}$ is limited to Cour $\in[0.2$, 4.0], with the Courant number step $\Delta$ Cour $=0.05$. The exponent $\bar{\alpha}$ was determined as follows: the functions $\mu^{+-}$(Cour) were fixed equal to unity, and for each Courant number in the range above, the values $0.25,0.50$ and 0.75 for the exponent $\bar{\alpha}$ were tested. The best value found was 0.50 which corresponds to the smallest mean square error between exact and numerical solutions. After determining the $\bar{\alpha}$ value, the next step was to determine the $\mu^{+/-}$(Cour) functions. By using $\bar{\alpha}=0.5$, for each Courant number fixed, was determined as being the best value for these functions those that gave the smallest mean square error between exact and numerical solutions. The final results are presented in the Fig. 3. We notice that a fast interpolation scheme to obtain the functions $\mu^{+/-}$(Cour) at any point inside the range can be easily determined.

\section{Numerical examples for one-dimensional case}

In this section we present two numerical examples for a onedimensional case. The operator obtained with the methodology here presented will be denoted by TDG + DC. The results are compared with exact solution, TDG method and with the method presented in [8], which will be denoted by TDG $+\mathrm{HH}$.

\subsection{Example 1 - Homogenous one-dimensional elastic bar}

The first example presented here considers a homogenous onedimensional elastic bar with one end fixed and the other loaded by an axial force, which represents a typical one-dimensional wave propagation problem. In this example both stresses and velocities present discontinuity on space and time. The properties of the bar are: length $L_{x}=4 \mathrm{~m}$, square cross section $0.04 \mathrm{~m}^{2}$, mass density $\rho=1 \mathrm{~kg} / \mathrm{m}^{3}$ and Young's modulus $E=1 \mathrm{~N} / \mathrm{m}^{2}$. A $200 \times 1$ mesh of quadratic Lagrangian space-time elements was used in each time-step. The force applied at the free end of the bar at initial time is a Heaviside of intensity $10^{-2} \mathrm{~N}$.

The results for velocities and stresses obtained for Courant numbers $0.57,1.03$ and 2.03, which correspond respectively to

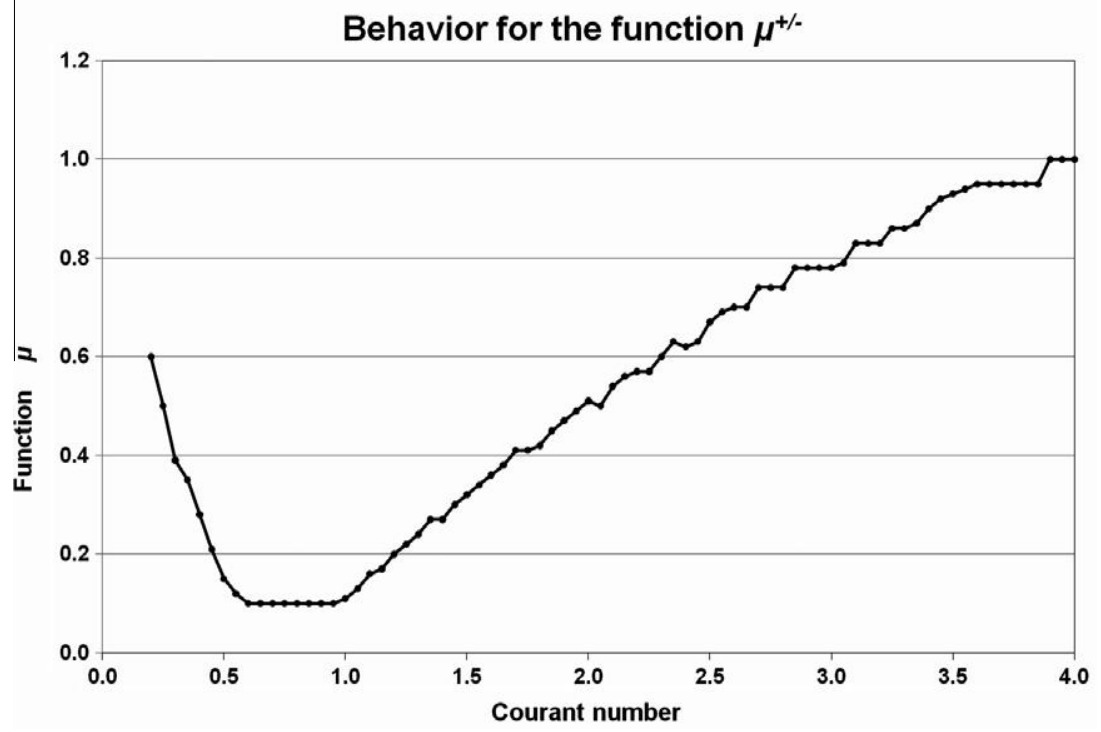

Fig. 3. Behavior of the function $\mu^{+/-}$(Cour) with Courant number. 
the following time steps $1.14 \times 10^{-2} \mathrm{~s}, 2.06 \times 10^{-2} \mathrm{~s}$ and $4.06 \times 10^{-2} \mathrm{~s}$, are depicted in Fig. 4. Figs. 4a, $4 \mathrm{c}$ and $4 \mathrm{e}$ show the behavior of the velocity at the tip of the bar along the time, while Figs. $4 \mathrm{~b}, 4 \mathrm{~d}$ and $4 \mathrm{f}$ show the behavior of the stress distribution at a specific time along the bar. It should be noted that only a small interval of the time and space are presented in those figures. The exact solution of this example can be found in [13].
Some remarks can be done about the behavior of the methods results. In all tests, the TDG method presented an overshoot and an undershoot close to the discontinuity, because this method does not control the second derivative. The other methods do not present these oscillations. For all ranges of Courant numbers, the proposed method (TDG + DC) is less dissipative than the TDG $+\mathrm{HH}$ method. The difference between the two methods is more

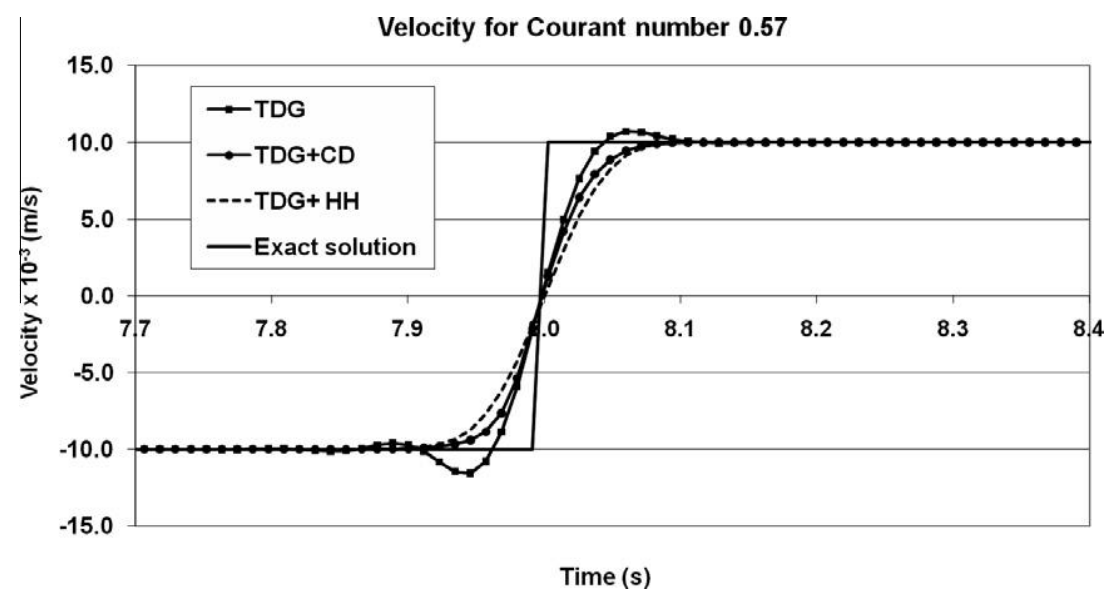

Fig. 4a. Velocity at the tip of the bar along the time for Courant number 0.57 .

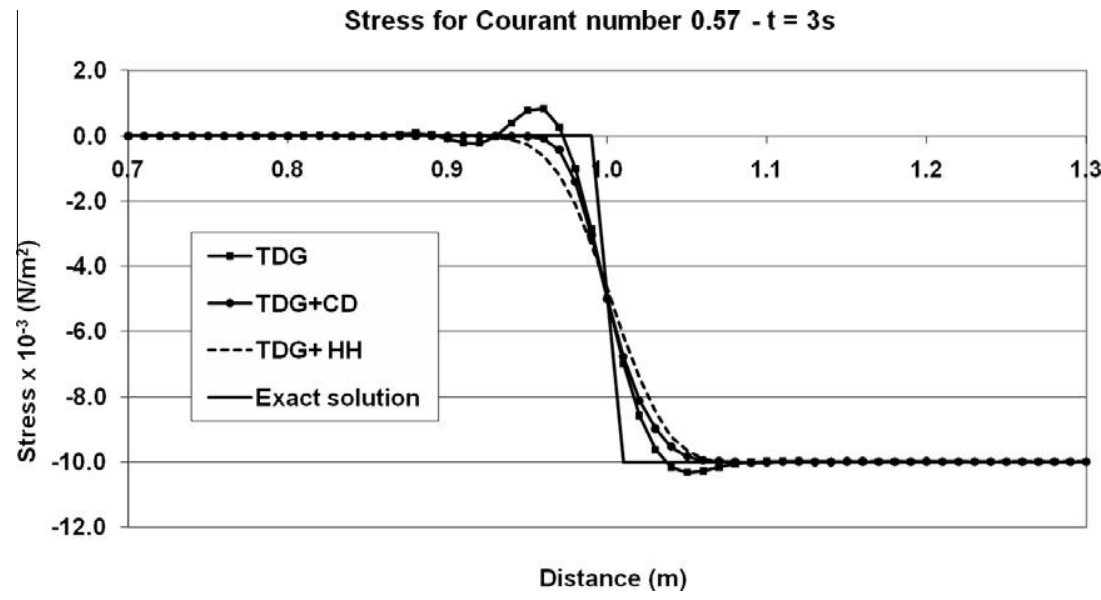

Fig. 4b. Stress distribution along the bar for Courant number 0.57 .

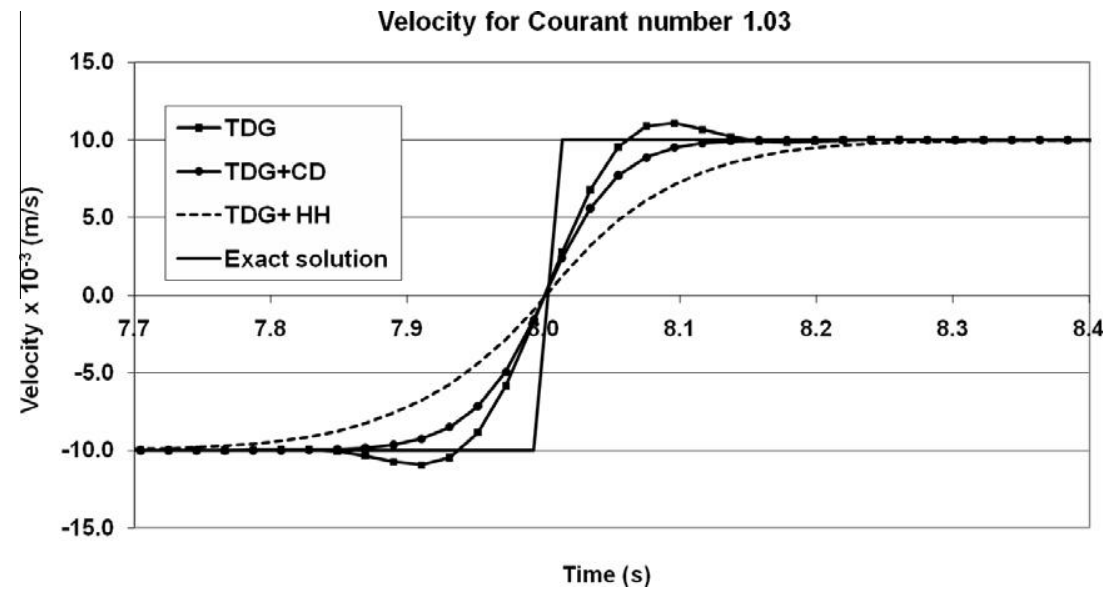

Fig. 4c. Velocity at the tip of the bar along the time for Courant number 1.03 . 


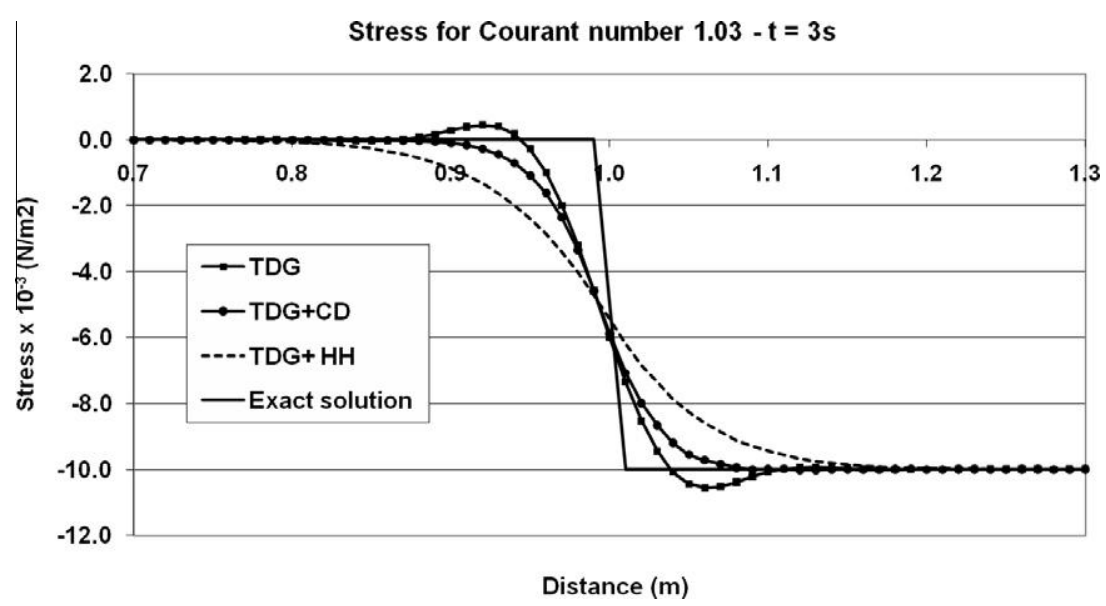

Fig. 4d. Stress distribution along the bar for Courant number 1.03.

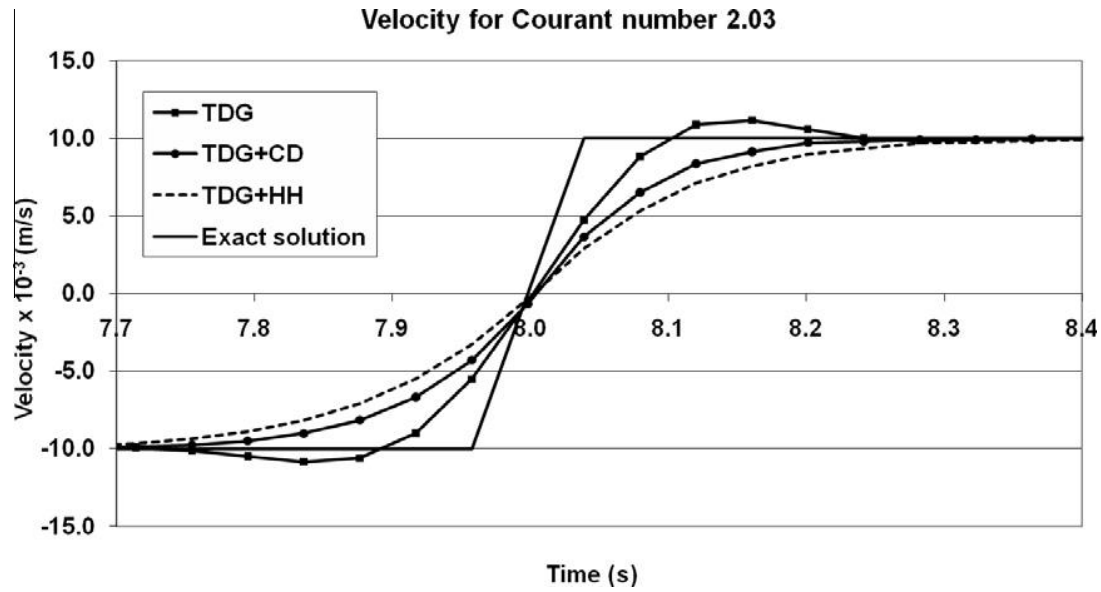

Fig. 4e. Velocity at the tip of the bar along the time for Courant number 2.03 .

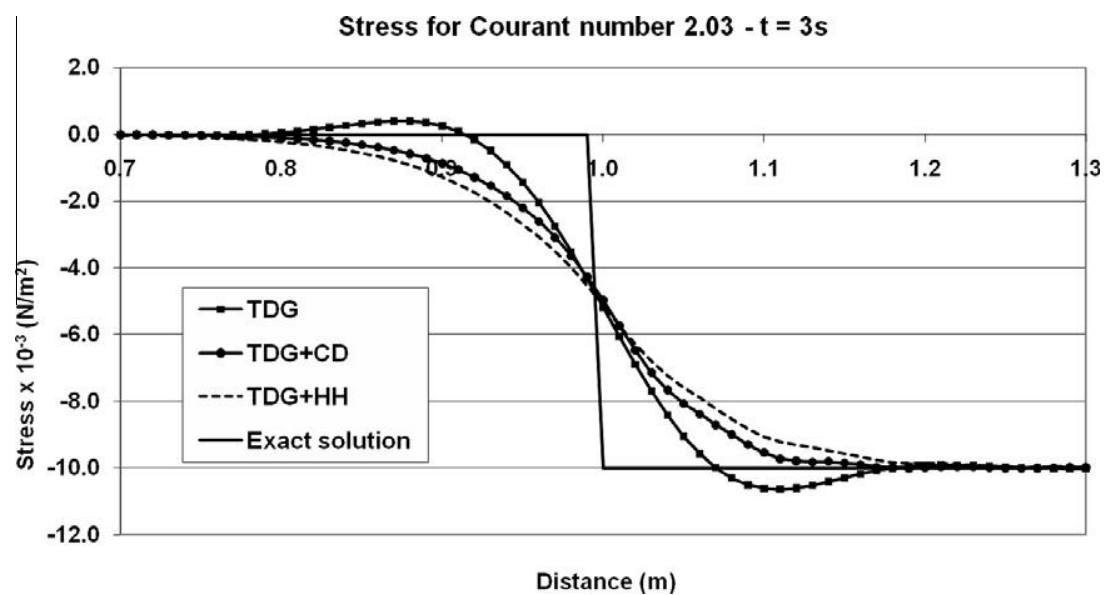

Fig. 4f. Stress distribution along the bar for Courant number 2.03 .

pronounced for Courant numbers close to 1 . We can observe from the results presented that the TDG + DC method does not present overshoots and undershoots. It should be noted that the methods TDG + DC and TDG + HH are non-linear; for the numerical tests, presented here, the number of iterations to achieve convergence of the proposed method was equal to two.

\subsection{Example 2 - Non-homogeneous one-dimensional elastic bar}

The second example analyzed considers a one-dimensional elastic bar consisting of two different homogenous domains. The material properties are: $L_{1}=2 \mathrm{~m}, E_{1}=4 \mathrm{~N} / \mathrm{m}^{2} ; c_{1}=2.0 \mathrm{~m} / \mathrm{s}$ and $L_{2}=2 \mathrm{~m}, E_{2}=1 \mathrm{~N} / \mathrm{m}^{2} ; c_{2}=1.0 \mathrm{~m} / \mathrm{s}$, and the square cross section is 
$0.04 \mathrm{~m}^{2}$ along the entire bar. A $200 \times 1$ mesh of quadratic Lagrangian elements was used in each time-step. The force applied at the free end of the bar has intensity of $10^{-2} \mathrm{~N}$ and short duration (only one time step) as depicted in Fig. 5. Fig. 6 shows results obtained for Courant numbers equal to $0.57,1.03$ and 2.03, which correspond respectively to the following time steps $1.14 \times 10^{-2} \mathrm{~s}$, $2.06 \times 10^{-2} \mathrm{~s}$ and $4.06 \times 10^{-2} \mathrm{~s}$.

Figs. $6 \mathrm{a}, 6 \mathrm{c}$ and $6 \mathrm{e}$ show the time history of the displacement at the tip of the bar, while the Figs. $6 \mathrm{~b}, 6 \mathrm{~d}$ and $6 \mathrm{f}$ correspond to the behavior of the stress distribution along the bar at a specific time. It should be noted that only a short interval of the time and space are presented in those figures.

Again, the proposed discontinuity-capturing operator does not present oscillations and we observe that for all ranges of Courant number, the proposed method (TDG + DC) is less dissipative than the TDG + HH method, and the difference between the two methods is more pronounced for Courant numbers close to 1. Again, the convergence of the method was achieved with two iterations.
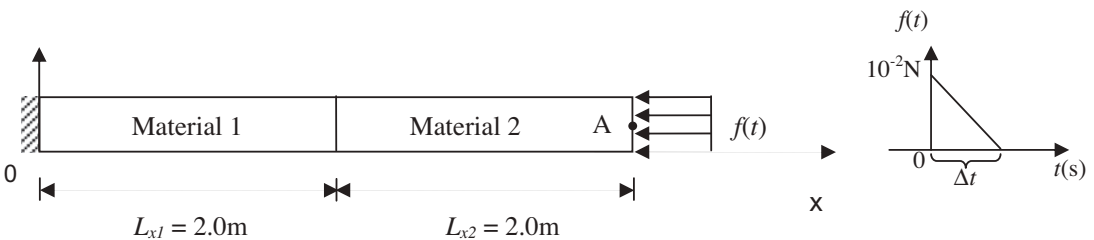

Fig. 5. Two material elastic bar.

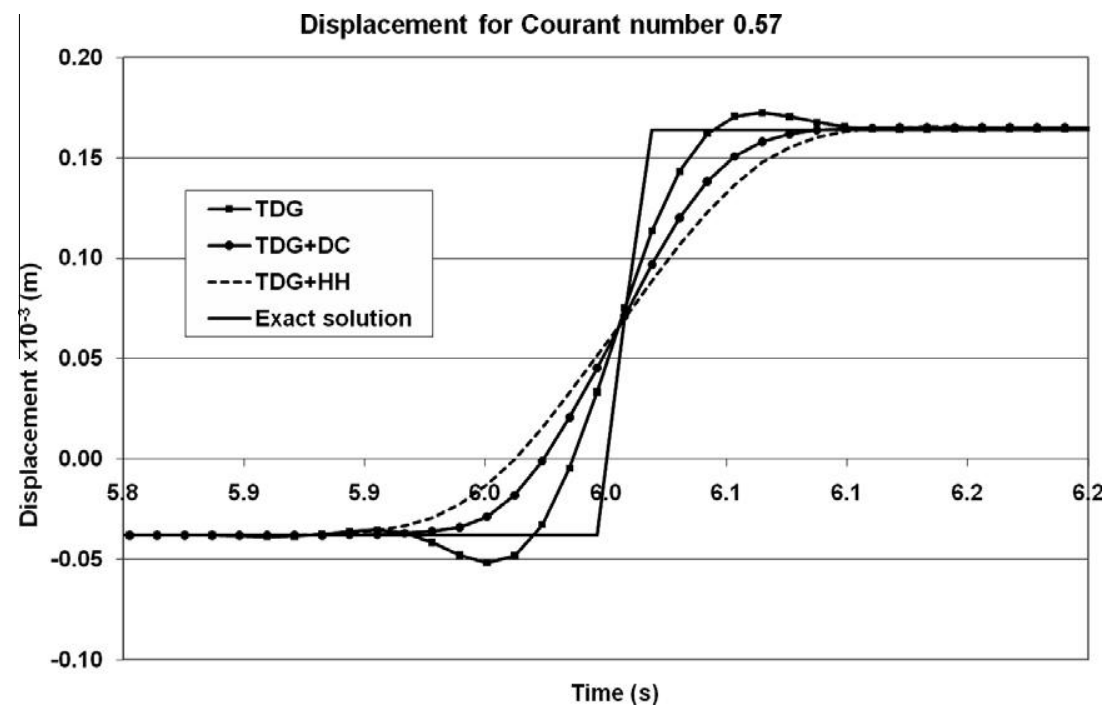

Fig. 6a. Displacement at the tip of the bar along the time for Courant number 0.57.

Stress for Courant number $0.57-\mathrm{t}=2.5 \mathrm{~s}$

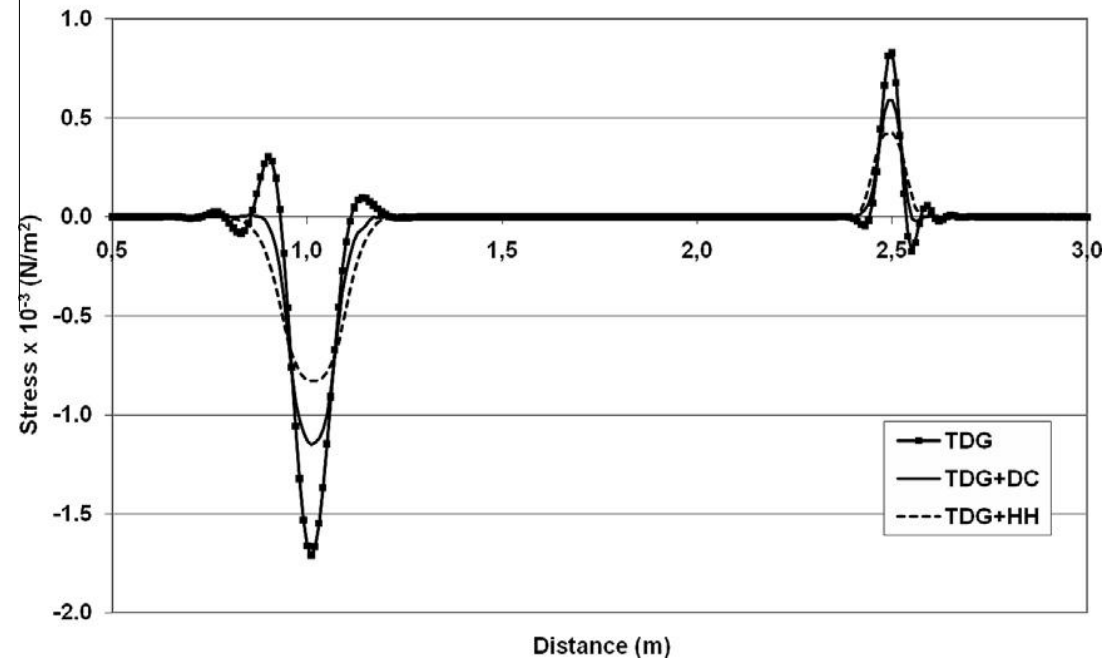

Fig. 6b. Stress distribution along the bar for Courant number 0.57 . 


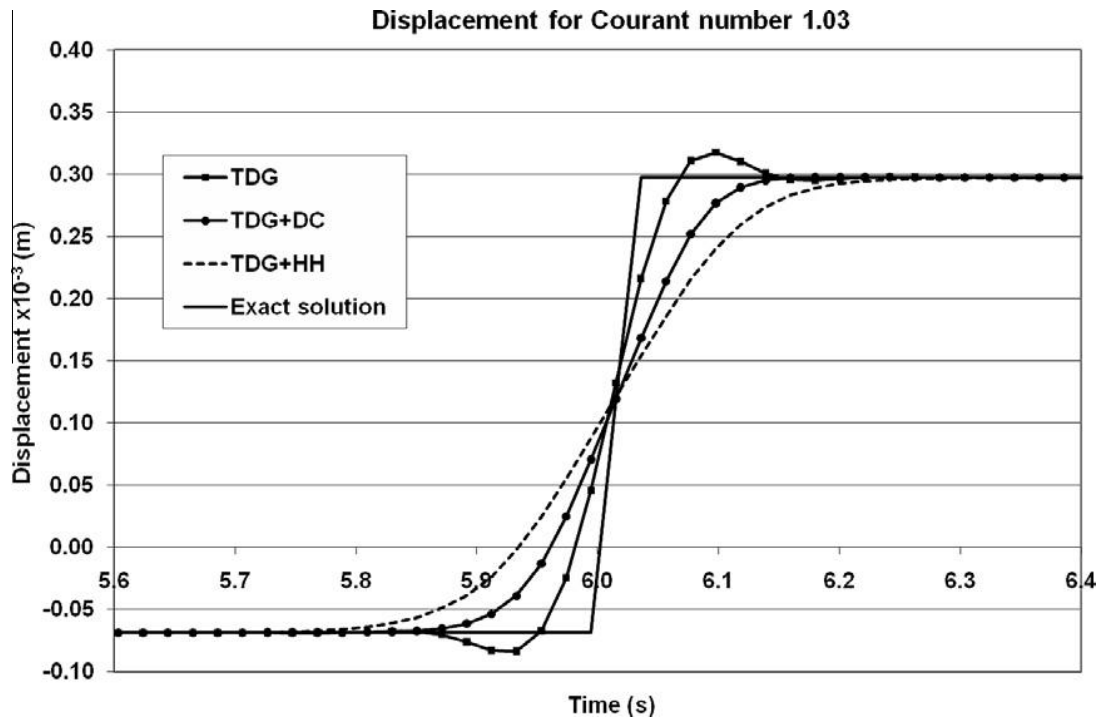

Fig. 6c. Displacement at the tip of the bar along the time for Courant number 1.03 .

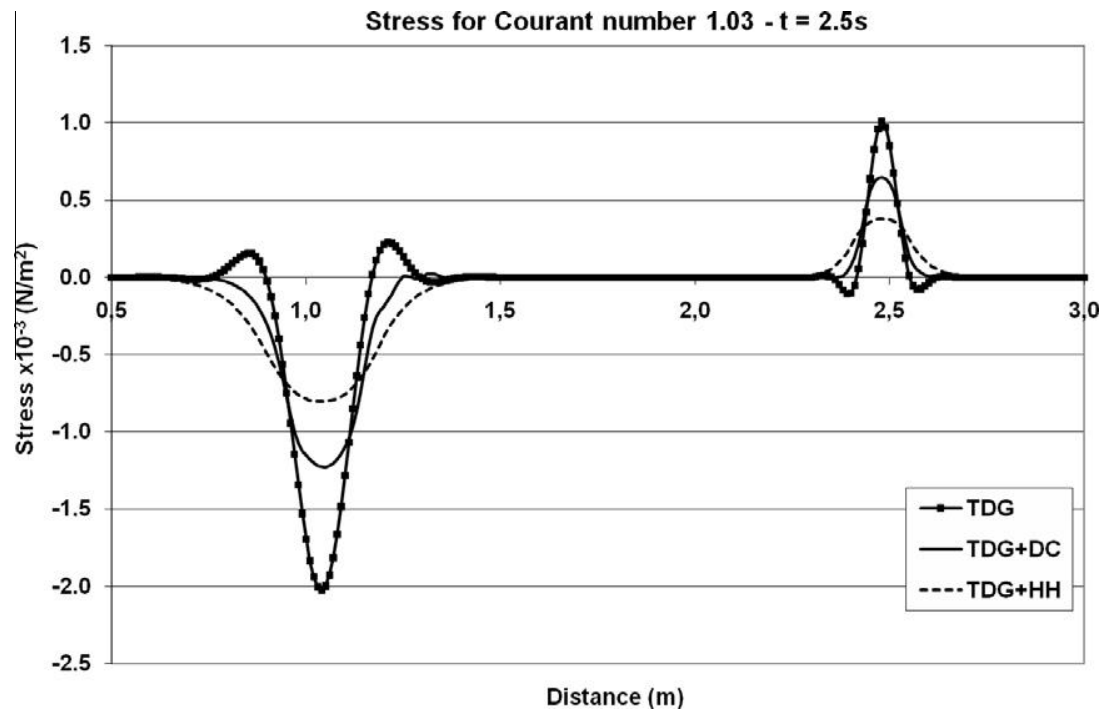

Fig. 6d. Stress distribution along the bar for Courant number 1.03 .

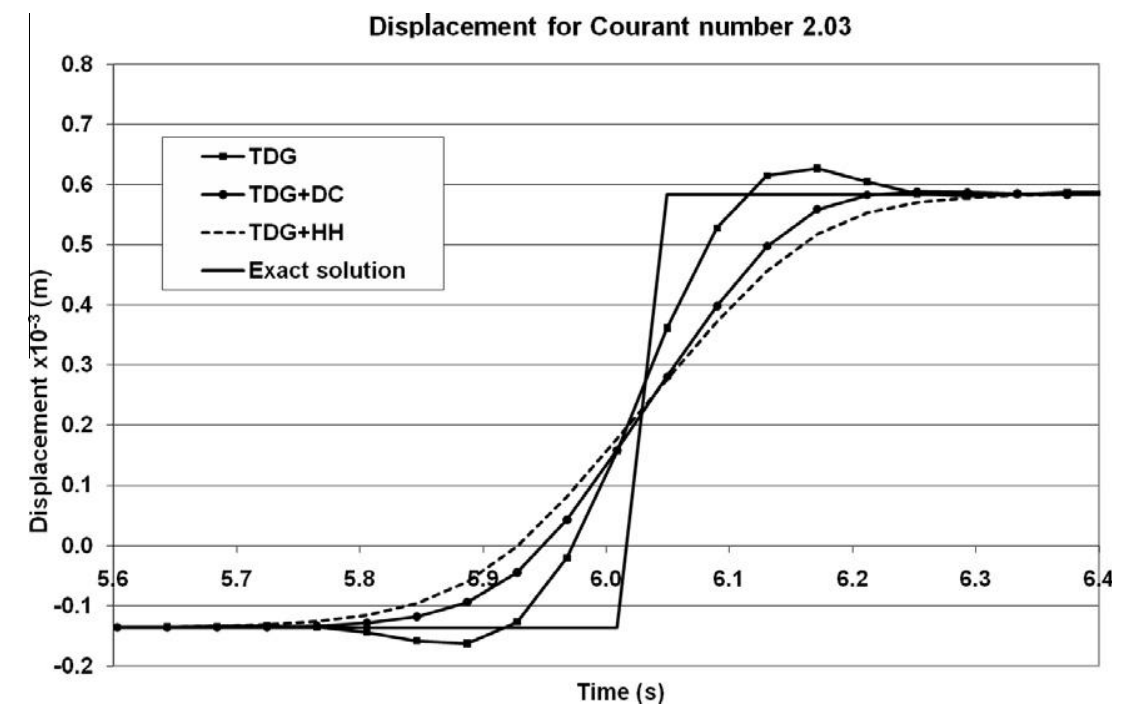

Fig. 6e. Displacement at the tip of the bar along the time for Courant number 2.03. 


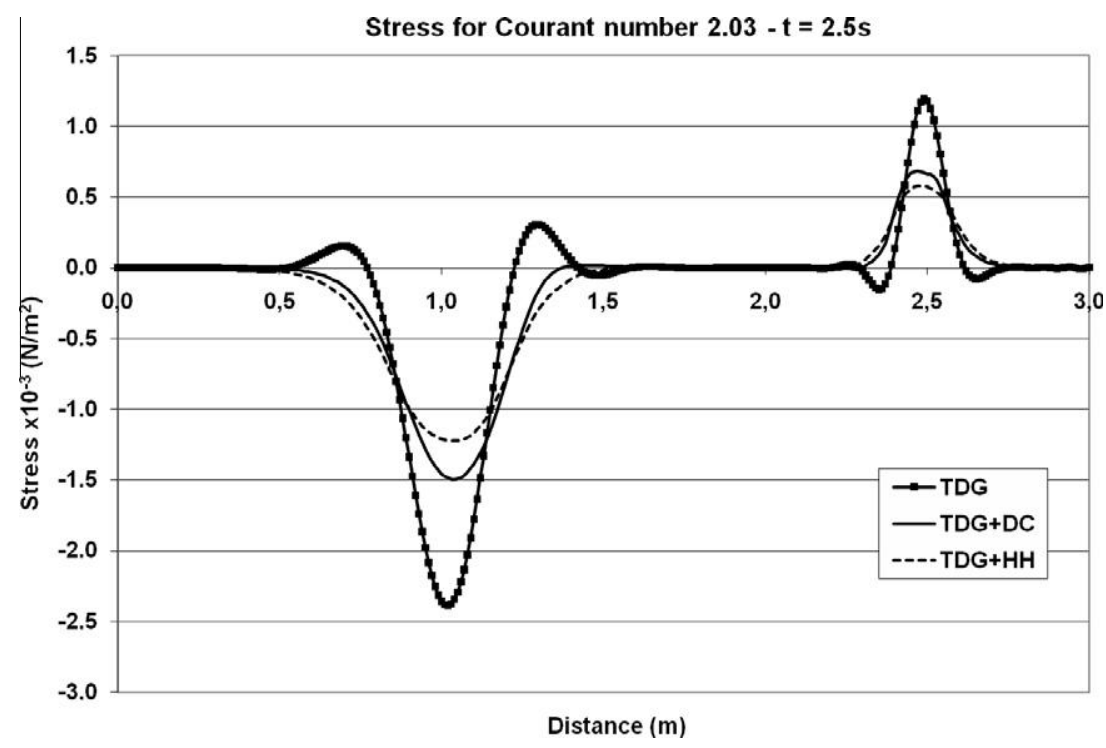

Fig. 6f. Stress distribution along the bar for Courant number 2.03 .

\section{Extension for the d-dimensional case}

In this section we propose an extension of the discontinuitycapturing operator for $d$-dimensional problems. However, the extension for the $d$-dimensional problem of the capturing operator is not trivial. The results obtained from a one-dimensional experiment, suggests that the stabilization parameter of the discontinuity-capturing operator is a function of Courant number. However, for problems of dimension greater than one, there are many possible choices to evaluate the Courant number for each direction. Based on numerical experiments, an optimal or quasi-optimal parameter was obtained for one-dimensional problems. However, numerical tests with quadrilateral elements using this parameter on all directions and with only one Courant number suggested: (a) Appropriated Courant numbers must be evaluated on each direction and, b) one reduction factor must multiply the onedimensional stabilization parameter for each direction in $d$-dimensional problems, otherwise, excessive dissipation can appear.

Numerical experiments with quadrilateral elements have indicated that an appropriate expression for the Courant number on the $i$-direction can be similar to that given in Section 4 and adopted as follows

$\operatorname{Cour}_{i}=\frac{\left(\frac{\left|C_{i i i j}\right|}{\rho}\right)^{1 / 2} \Delta t}{h_{e, i}}$,

where $h_{e, i}$ is as given in Section 4, Eq. (31). The same numerical experiments also have indicated that an appropriate reduction factor must possess information concerning the distortion of the element.

By noting that the Jacobean matrix possesses intrinsically this information, we propose the following expression for the reduction factor on the $i$-direction

$F_{\text {red }, i}=F_{\text {red }, 0} \frac{\left(H_{e, J}\right)_{i}}{\left\|H_{e, J}\right\|}$,

with $H_{e J}$ given by

$H_{e, J}=\mathbf{J}\left(\begin{array}{c}h_{e, 1} \\ : \\ h_{e, d}\end{array}\right)$,

where $\mathbf{J}$ is the Jacobean matrix, $\left(H_{e J}\right)_{i}$ is the $i$-order component of vector $H_{J}$ and $F_{r e d, 0} \leqslant 1$ is a dimensionless number.
Extensive numerical tests with meshes of quadrilateral elements were made to $F_{r e d, 0}=0.25, F_{r e d, 0}=0.50$ and $F_{r e d, 0}=0.75$. Despite the low sensitivity for this range of values of $F_{\text {red, } 0}$, was observed that the best results for all ranges of Courant number were obtained with $F_{r e d, 0}=0.50$.

Preliminary tests with various Courant numbers using triangular meshes obtained from regular meshes with quadrilateral elements, making each quadrilateral into two triangles, have been made with $F_{\text {red, } 0}=0.25, F_{\text {red }, 0}=0.50$ and $F_{\text {red }, 0}=0.75$. Again, there was little sensitivity and the best results were obtained for $F_{\text {red }}$ ${ }_{0}=0.50$. However it would be good to repeat these tests for triangular unstructured meshes in order to confirm this result.

By using the functions $\mu^{+/-}\left(\right.$Cour $\left._{i}\right)$ obtained for the one-dimensional case, one can define the factors $F_{1}^{+/-}\left(\right.$Cour $\left._{1}\right), \ldots, F_{d}^{+/-}\left(\right.$Cour $\left._{d}\right)$ to each direction $x_{1}, \ldots x_{i}, \ldots x_{d}$ as follows

$F_{i}^{+/-}\left(\operatorname{Cour}_{i}\right)=F_{\text {red }, i} \times \mu^{+/-}\left(\right.$Cour $\left._{i}\right)$.

By considering the cross factors $F_{m k}^{+/-}$and $F_{t}^{+/-}$given by the expressions that follow

$F_{m k}^{+/-}=\left(F_{m}^{+/-}\left(\operatorname{Cour}_{m}\right) \times F_{k}^{+/-}\left(\operatorname{Cour}_{k}\right)\right)^{1 / 2}$

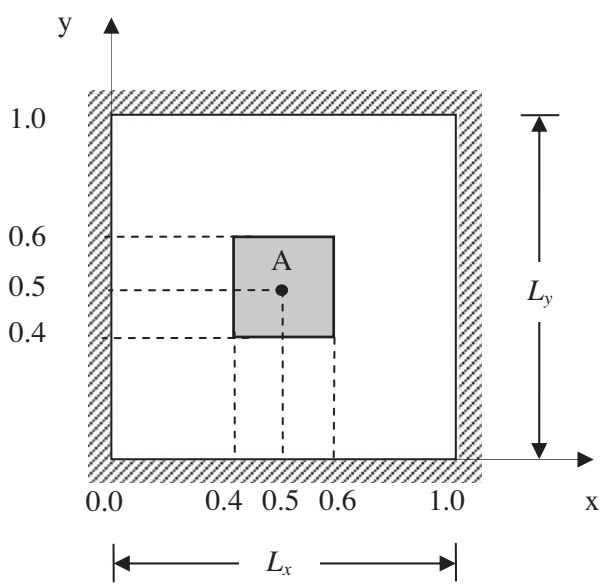

Fig. 7. Square membrane under prescribed initial velocity, over the area A, superior view. 
and

$F_{t}^{+/-}=\operatorname{Max}\left\{F_{1}^{+/-}\left(\operatorname{Cour}_{1}\right), \ldots, F_{d}^{+/-}\left(\operatorname{Cour}_{d}\right)\right\}$

then for each $i \in\{1, \ldots, d\}$ a function $\mathbf{W}_{i}\left(\mathbf{W}^{h}\right)$ can be defined for the $d$-dimensional case as follows

$\mathbf{W}_{i}\left(\mathbf{W}^{h}\right)=\left\{\begin{array}{l}\mathbf{W}_{i,-}\left(\mathbf{W}^{h}\right), \text { if } \operatorname{Cour}_{i}<1 \\ \mathbf{W}_{i,+}\left(\mathbf{W}^{h}\right), \text { if } \operatorname{Cour}_{i} \geqslant 1\end{array}\right.$,

$\mathbf{W}_{i,-}\left(\mathbf{W}^{h}\right)=\left(\mathbf{W}_{i,-}^{1}\left(\mathbf{W}^{h}\right), \mathbf{W}_{i,-}^{2}\left(\mathbf{W}^{h}\right), F_{t}^{-} \times\left(\operatorname{Cour}_{i}\right)^{4} \frac{1}{\sqrt{\gamma_{i}}} \frac{\partial^{2} W_{i}^{h}}{\partial t^{2}}\right)$,

$\mathbf{W}_{i,-}^{1}\left(\mathbf{W}^{h}\right)=\left(F_{11}^{-} \delta_{i 111} \frac{\partial^{2} W_{1}^{h}}{\partial x_{1}^{2}}, \ldots, F_{j l}^{-} \delta_{i j k l} \frac{\partial^{2} W_{k}^{h}}{\partial x_{j} \partial x_{l}}, \ldots, F_{d d}^{-} \delta_{i d d d} \frac{\partial^{2} W_{d}^{h}}{\partial x_{d}^{2}},\right)$

$\mathbf{W}_{i,-}^{2}\left(\mathbf{W}^{h}\right)=\left(F_{11}^{-} \frac{\delta_{i 111}^{1}}{\sqrt{\theta_{i}}} \frac{\partial W_{1}^{h}}{\partial x_{1}}, \ldots, F_{j l}^{-} \frac{\delta_{i j k l}^{j}}{\sqrt{\theta_{i}}} \frac{\partial W_{k}^{h}}{\partial x_{l}}, \ldots, F_{d d}^{-} \frac{\delta_{i d d d}^{d}}{\sqrt{\theta_{i}}} \frac{\partial W_{d}^{h}}{\partial x_{d}},\right)$

$\mathbf{W}_{i,+}\left(\mathbf{W}^{h}\right)=\left(\mathbf{W}_{i,+}^{1}\left(\mathbf{W}^{h}\right), \mathbf{W}_{i,+}^{2}\left(\mathbf{W}^{h}\right), F_{t}^{+} \frac{1}{\sqrt{\gamma_{i}}} \frac{\partial^{2} W_{i}^{h}}{\partial t^{2}}\right)$,

$\mathbf{W}_{i,+}^{1}\left(\mathbf{W}^{h}\right)=\left(\operatorname{Cour}_{i}\right)^{-4}\left(F_{11}^{+} \delta_{i 111} \frac{\partial^{2} W_{1}^{h}}{\partial x_{1}^{2}}, \ldots, F_{j l}^{+} \delta_{i j k l} \frac{\partial^{2} W_{k}^{h}}{\partial x_{j} \partial x_{l}}, \ldots, F_{d d}^{+} \delta_{i d d d} \frac{\partial^{2} W_{d}^{h}}{\partial x_{d}^{2}},\right)$

$\mathbf{W}_{i,+}^{2}\left(\mathbf{W}^{h}\right)=\left(\operatorname{Cour}_{i}\right)^{-4}\left(F_{11}^{+} \frac{\delta_{i 111}^{1}}{\sqrt{\theta_{i}}} \frac{\partial W_{1}^{h}}{\partial x_{1}}, \ldots, F_{j l}^{+} \frac{\delta_{i j k l}^{j}}{\sqrt{\theta_{i}}} \frac{\partial W_{k}^{h}}{\partial x_{l}}, \ldots, F_{d d}^{+} \frac{\delta_{i d d d}^{d}}{\sqrt{\theta_{i}}} \frac{\partial W_{d}^{h}}{\partial x_{d}},\right)$

where $W_{i}^{h}$ is the component of $i^{\text {th }}$ order of the vector $\mathbf{W}^{h}$.

\section{Numerical example for two-dimensional case}

In this section we present one numerical example for a twodimensional case. The operator obtained with the methodology here presented will be denoted by TDG + DC. The results are compared with the exact solution found in [13], classical Newmark's method and TDG method.

\subsection{Example 3 - Transverse motion of quadrangular membrane} under prescribed initial velocity

The last example considers the transverse motion of a square membrane with initial velocity $1 \mathrm{~m} / \mathrm{s}$ applied transversely over the shaded area $\mathrm{A}(0.2 \mathrm{~m} \times 0.2 \mathrm{~m})$ and zero displacements prescribed over all boundary and zero initial displacement prescribed over the domain (see Fig. 7). The length of each side of the membrane is equal to $1 \mathrm{~m}$ and the wave propagation velocity is $1 \mathrm{~m} / \mathrm{s}$. The variable chosen to verify accuracy is the velocity time history at the center of the membrane. Due to the symmetry of the problem, only the one quarter of the membrane needs to be discretized. Each space-time slab of the one quarter was discretized with 1600 hexahedral elements of twenty-seven nodes. In this example, the methods considered were classical Newmark with parameters ( $\delta=0.50$ and $\alpha=0.25$ ) as given in [14], TDG and TDG + DC. The results are compared with the exact solution. The Courant numbers considered were $0.50,1.00$ and 1.75 which correspond respectively to the time steps $6.25 \times 10^{-3} \mathrm{~s}$, $1.25 \times 10^{-2} \mathrm{~s}$, and $2.19 \times 10^{-2} \mathrm{~s}$. The results are presented in the Fig. 8.

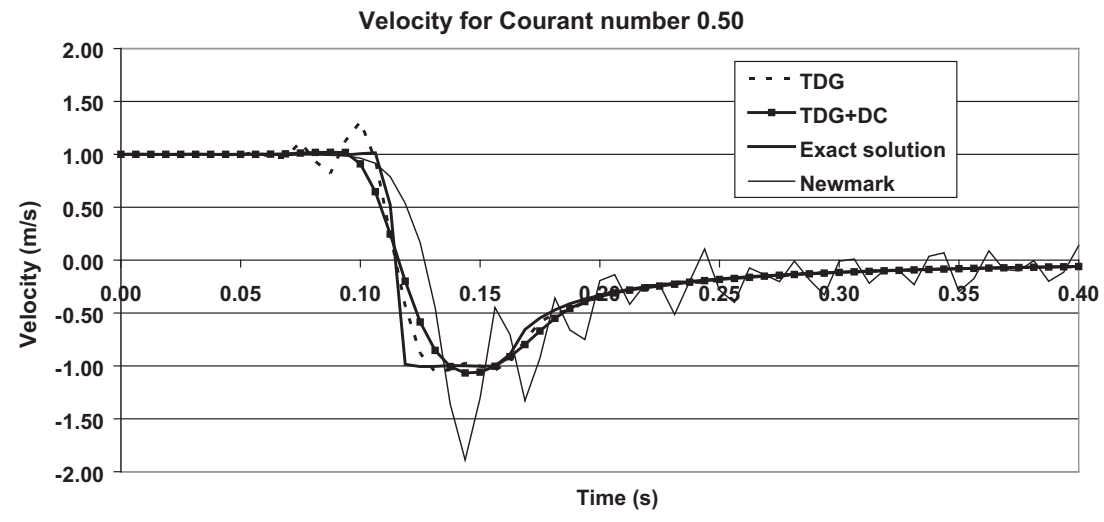

Fig. 8a. Velocity for Courant number 0.50 .

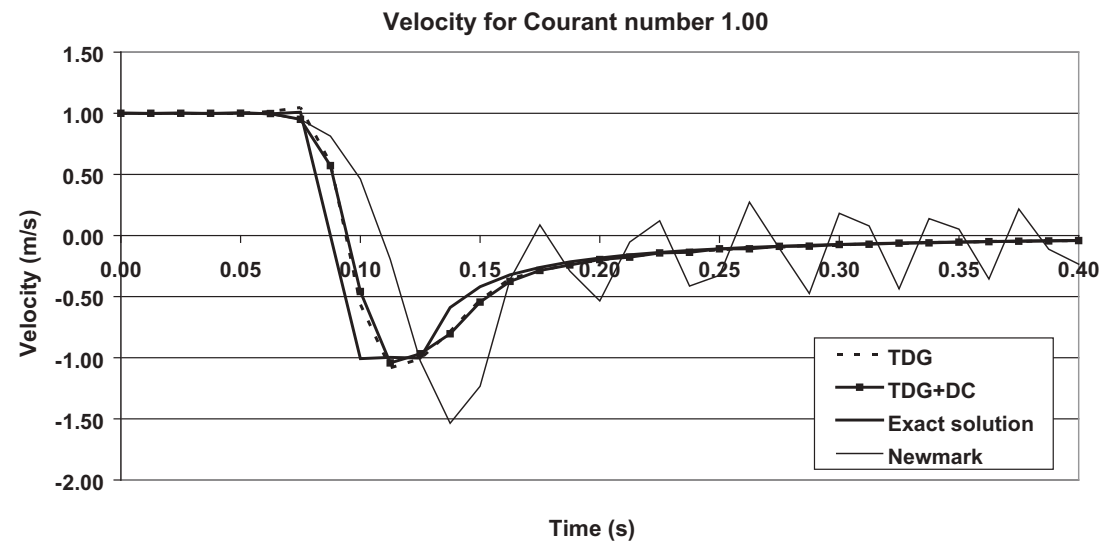

Fig. 8b. Velocity for Courant number 1.00 . 


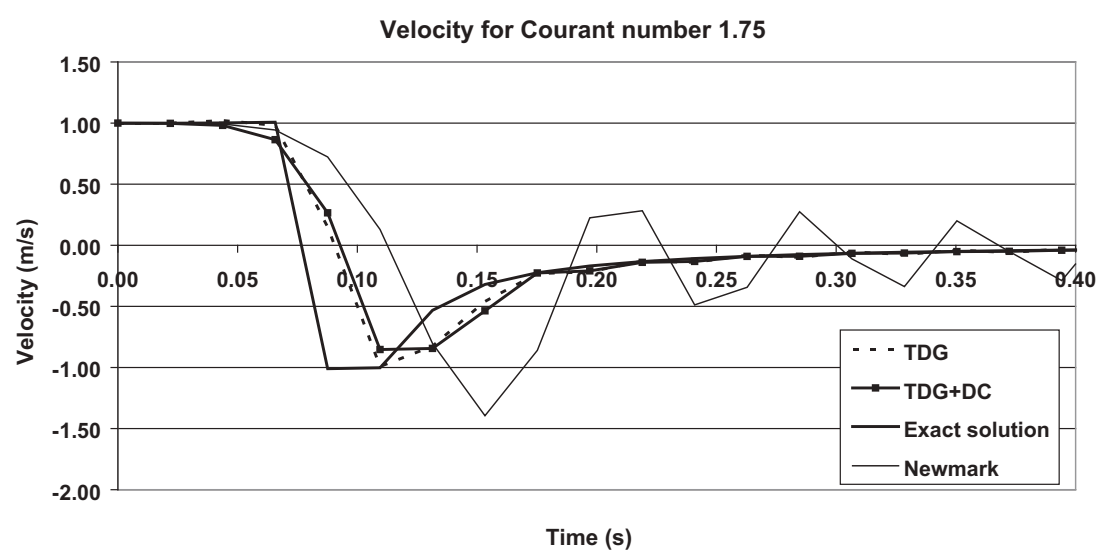

Fig. 8c. Velocity for Courant number 1.75 .

This example uses the extension proposed to 2D problems in previous section to the discontinuity-capturing operator. As well as the one-dimensional examples, this case presents discontinuity in the velocity. As can be seen in Fig. 8, the (TDG + DC) method has dissipation slightly higher that the TDG method but without spurious oscillations, overshoots and undershoots.

Remark. As can be observed in the results presented, the Newmark's method has spurious oscillations in the presence of high gradients (on velocities and stresses) while the TDG method does not exhibit strong spurious oscillations but only presents overshoots and undershoots, and has equivalent dissipation or less than the Newmark's method. These observations apply to other traditional methods as well as to all methods of the Newmark's family. Therefore, the TDG method can be the basis for evaluating the performance of the (TDG + DC) method presented in this paper.

\section{Conclusions}

In this paper we present a general methodology to obtain families of discontinuity capturing operators for elastodynamics. This methodology is based on the work developed in [12] to diffusion-convection problems, and inspired in the operator presented in [7]. The operators are indicated for problems with discontinuities. The methodology was applied to problems with discontinuities in the time and in the space and for all ranges of Courant numbers presented the operator proposed here was less diffusive than the operator presented in [7]. The tests show that the proposed method (TDG + DC) does not present overshoots and undershoots, because the discontinuity-capturing operator controls the second order derivatives of the displacement. This difference is more pronounced for Courant numbers close to 1 .

In addition it is proposed an extension for $d$-dimensional problems. It should be noted that the proposed extension presented in Section 9 is not definitive, but a possible extension for $d$-dimensional case. Robust tests with distorted meshes including those with triangular elements must be done, in such a way to validate or suggest modifications on the proposed extension.

The TDG + DC and TDG + HH are non-linear methods, and the numerical simulations with the proposed discontinuity-capturing operator give a clear indication that two iterations are sufficient to achieve convergence of the solution, more iterations lead to no improvement.

\section{Acknowledgment}

The authors gratefully acknowledge the financial support of CAPES (Coordenação de Aperfeiçoamento de Pessoal de Nivel Superior) and the Brazilian Research Funding Agencies (CNPq).

\section{References}

[1] J.T. Oden, A general theory of finite elements Part II, International Journal for Numerical Methods in Engineering 1 (1969) 247-259.

[2] J.H. Argyris, D.W. Scharpf, Finite elements in time and space, Nuclear Engineering and Design 10 (1969) 456-464.

[3] I. Fried, Finite element analysis of time-dependent phenomena, AIAA Journal 13 (1969) 1154-1157.

[4] W.H. Reed, T.R. Hill, Triangular Mesh Methods for the Neutron Transport Equation, Report LA-UR-73-479, 1973, Los Alamos Scientific Laboratory, Los Alamos.

[5] P. Lesaint, P.A. Raviart, On a finite element method for solving the neutron transport equation, in: C. de Boor (Ed.), Mathematical Aspects of Finite Elements in Partial Differential Equations, Academic Press, New York, 1974, pp. 89-123.

[6] T.J.R. Hughes, G.M. Hulbert, Space-time finite element methods for elastodynamics: formulations and error estimates, Computer Methods in Applied Mechanics and Engineering 66 (1988) 339-363.

[7] G.M. Hulbert, T.J.R. Hughes, Space-time finite element methods for secondorder hyperbolic equations, Computer Methods in Applied Mechanics and Engineering 84 (1990) 327-348.

[8] G.M. Hulbert, Discontinuity-capturing operators for elastodynamics, Computer Methods in Applied Mechanics and Engineering 96 (1992) 409-426.

[9] V. Jonh, P. Knobloch, On spurious oscillations at layers diminishing (SOLD) methods for convection-diffusion equations: Part I - A review, Computer Methods in Applied Mechanics and Engineering 196 (2007) 2197-2215.

[10] X.D. Li, N.E. Wiberg, Implementation and adaptivity of a space-time finite element method for structural dynamics, Computer Methods in Applied Mechanics and Engineering 156 (1998) 211-229.

[11] E.G. Dutra do Carmo, A.C. Galeão, A consistent formulation of the finite element method to solve convective-diffusive transport problems, Brazilian Journal of Mechanical Sciences 4 (1986) 309-340.

[12] A.C. Galeão, E.G. Dutra do Carmo, A consistent approximate upwind petrovgalerkin method for convection-dominated problems, Computer Methods in Applied Mechanics and Engineering 68 (1988) 83-95.

[13] W.J. Mansur, A Time-Stepping Technique to Solve Wave Propagation Problems Using the Boundary Element Method, Ph. D. Thesis, University of Southampton, England, 1983.

[14] K.J. Bathe, Finite Element Procedures, Prentice-Hall, Englewood Cliffs, New Jersey, 1996. 